\title{
Claudin-1 Is a Metastasis Suppressor and Correlates with Clinical Outcome in Lung Adenocarcinoma
}

\author{
Yu-Chih Chao ${ }^{1}$, Szu-Hua Pan ${ }^{1}$, Shuenn-Chen Yang ${ }^{2}$, Sung-Liang $\mathrm{Yu}^{3}$, Ting-Fang Che ${ }^{4}$, Chung-Wu Lin ${ }^{5}$, \\ Mu-Shiun Tsai ${ }^{5}$, Gee-Chen Chang ${ }^{6}$, Che-Hsiang $\mathrm{Wu}^{2}$, Yi-Ying $\mathrm{Wu}^{1}$, Yung-Chie Lee ${ }^{7}$, Tse-Ming Hong ${ }^{2,8,9}$, \\ and Pan-Chyr Yang 2,8, 10,*
}

\begin{abstract}
${ }^{1}$ Graduate Institute of Life Sciences, National Defense Medical Center, Taipei, Taiwan; ${ }^{2}$ Institute of Biomedical Sciences, Academia Sinica, Taipei, Taiwan; ${ }^{3}$ Department of Clinical Laboratory Sciences and Medical Biotechnology, College of Medicine; ${ }^{4}$ Graduate Institute of Molecular Medicine, College of Medicine; ${ }^{5}$ Department of Pathology and Graduate Institute of Pathology, ${ }^{8}$ National Taiwan University Center of Genomic Medicine, Taipei, Taiwan, ${ }^{7}$ Department of Surgery; ${ }^{10}$ Department of Internal Medicine, National Taiwan University Hospital and National Taiwan University College of Medicine, Taipei, Taiwan; ${ }^{6}$ Division of Chest Medicine, Department of Internal Medicine, Taichung Veterans General Hospital, Taichung, Taiwan; ${ }^{9}$ Institute of Clinical Medicine, National Cheng Kung University, Tainan, Taiwan
\end{abstract}

Rationale: Claudin (CLDN)-1, a key component of tight junction complexes, was down-regulated in human lung adenocarcinomas. Objectives: To investigate the clinical significance of CLDN1 expression in patients with lung adenocarcinoma and its role in cancer invasion and metastasis.

Methods: We examined the CLDN1 mRNA expression in tumor specimens from 64 patients with lung adenocarcinoma and protein expression by immunohistochemistry in an independent cohort of 67 patients with lung adenocarcinoma. CLDN1 functions in cancer cell migration, invasion, and metastatic colonization were studied by overexpression and knockdown of CLDN1. Affymetrix microarrays were performed to identify gene expression changes associated with CLDN1 overexpression.

Measurements and Main Results: We found that low-CLDN1 mRNA expression had shorter overall survival $(P=0.027$, log-rank test) in 64 patients with lung adenocarcinoma, and we confirmed by immunohistochemistry that low CLDN1 expression had shorter overall survival ( $P=0.024$, log-rank test) in an independent cohort of 67 patients with lung adenocarcinoma. Overexpression of CLDN1 inhibited cancer cell dissociation in time-lapse imaging of wound healing, and suppressed cancer cell migration, invasion, and metastasis. Knockdown CLDN1 expression increased cancer cell invasive and metastatic abilities. Affymetrix microarrays identified a panel of genes altered by CLDN1 overexpression. CLDN1 increased expressions of cancer invasion/metastasis suppressors (e.g., connective tissue growth factor [CTGF], thrombospondin 1 [THBS1], deleted in liver cancer 1 [DLC1], occludin [OCLN], zona occludens 1 [ZO-1]) and suppressed expressions of invasion/metastasis enhancers (e.g., secreted phosphoprotein 1 [SPP1], cut-like homeobox 1 [CUTL1], transforming growth factor alpha [TGF- $\alpha$ ], solute carrier family 2 [faciliated glucose transporter] member 3 [SLC2A3], placental growth factor [PGF]), supporting a role for CLDN1 as an invasion and metastasis suppressor.

Conclusions: CLDN1 is a cancer invasion/metastasis suppressor. CLDN1 is also a useful prognostic predictor and potential drug treatment target for patients with lung adenocarcinoma.

Keywords: tight junctions; cell-cell adhesion; invasion

(Received in original form March 24, 2008; accepted in final form September 11, 2008) This work was supported by National Science Council (Taiwan) grant NSC952314-B002-119-MY3 (T.-M.H).

* Correspondence and requests for reprints should be addressed to Pan-Chyr Yang, M.D., Ph.D., Department of Internal Medicine, National Taiwan University College of Medicine, No. 7, Chung Shan South Road, Taipei, Taiwan. E-mail: pcyang@ntu.edu.tw

This article has an online supplement, which is accessible from this issue's table of contents at www.atsjournals.org

Am J Respir Crit Care Med Vol 179. pp 123-133, 2009

Originally Published in Press as DOI: 10.1164/rccm.200803-4560C on September 11, 2008 Internet address: www.atsjournals.org

\section{AT A GLANCE COMMENTARY}

Scientific Knowledge on the Subject

Loss of tight junction function may lead to loss of cell-cell adhesion and promote tumor metastasis. Claudin-1 (CLDN1), a key component of tight junctions, is downregulated in lung adenocarcinoma, but its role in cancer progression of lung adenocarcinoma is unknown.

What This Study Adds to the Field

CLDN1 inhibits lung adenocarcinoma cells from dissociating from each other and regulates cancer cell migration, invasion and metastasis. CLDN1 may be an invasion/ metastasis suppressor as well as a useful prognostic biomarker and potential therapeutic target.

Epithelial cells adhere tightly to their neighbors through a specialized junctional complex, including tight junctions (TJs), adherens junctions, and desmosomes. Cell-cell adhesion is important in maintaining epithelial morphology and tightly linked to cell proliferation and migration (1). During metastasis, cancer cells undergo a number of distinct steps. The initial step, which involves disruption of cell-cell junctions with concomitant changes in the expression of junctional complex proteins, is a hallmark of cancer invasion and metastasis (2). The loss of TJ function in carcinomas is regarded as an important step in the process that leads to loss of cell-cell adhesion and tumor metastasis (3). Losses of TJ molecules, such as zona occludens 1 (ZO-1) and occludin (OCLN), have been reported to correlate with tumor progression $(4,5)$.

TJs, which are localized to the apical end of lateral membrane surfaces of epithelial cells, are essential intercellular junctions, indispensable for the establishment of compositionally distinct compartments in multicellular organisms (3). Proteins that are integral components of TJ complexes include occludin, claudins, and junctional adhesion molecules. Claudins are known to be key components of TJs and form the backbone of TJ strands (6).

Claudins, which belong to a family consisting of at least 24 members, contain four transmembrane domains, two extracellular loops, and a carboxyl intracellular tail. The C-terminal sequences of claudins all share a PSD-95/Dlg/ZO-1 (PDZ) binding motif that is required for binding to the $\mathrm{PDZ}$ domains of other proteins, including ZO-1, ZO-2, ZO-3, multiple PDZ domain protein (MUPP1), and PALS1-associated TJ protein (PATJ) (7). These protein-protein interactions provide links to the actin cytoskeleton, and also act as adapters, serving to recruit cytosolic molecules that are potentially involved in mediating signaling to and from TJs. 
A

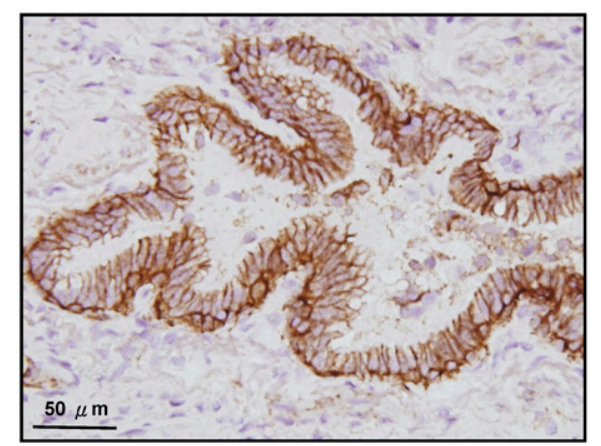

C

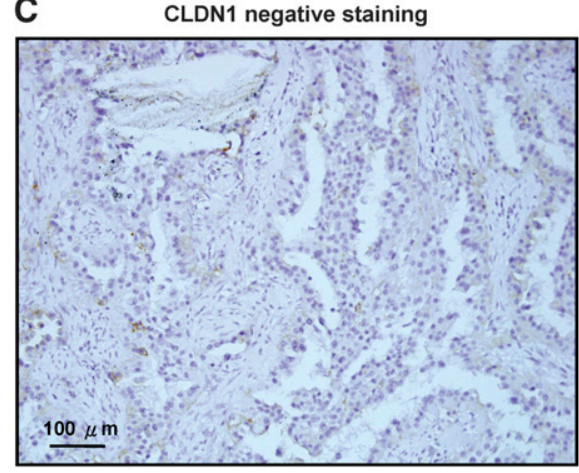

B
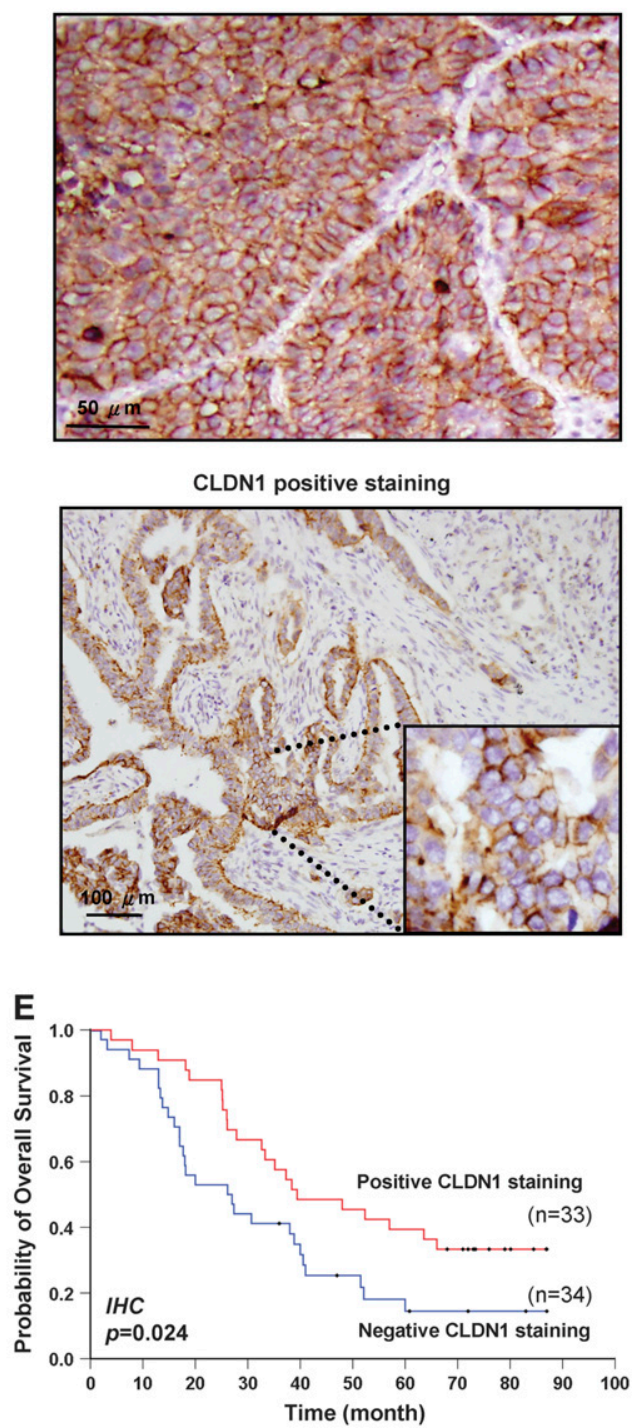

Figure 1. Claudin-1 (CLDN1) expression levels correlate with the survival of patients with lung adenocarcinoma. $(A)$ Immunohistochemical staining of CLDN1 expression in the lung tissue of an autopsy specimen. CLDN1 was expressed in the cell-cell junction of small airway columnar epithelial cells and basal cells. (B) High CLDN1 expression was detected in lung squamous cell carcinomas in immunohistochemical staining. (C) Representative images of negative and positive CLDN1 immunohistochemical staining in tumor specimens from patients with lung adenocarcinoma. (D) Kaplan-Meier estimates of overall survival in 64 patients with surgically resected lung adenocarcinoma: relationship to CLDN1 mRNA expression. CLDN1 mRNA levels were measured by real-time quantitative reverse transcriptase-polymerase chain reaction (RT-PCR) in primary tumor specimens. The relative amount of tissue CLDN1 mRNA was standardized against the geometric mean of TBP and glyceraldehyde 3-phosphate dehydrogenase mRNAs. The median CLDN1 value was used to divide patients into high CLDN1- and low CLDN1expressing groups. Red line, patients with high CLDN1 expression; blue line, patients with low CLDN1 expression. $P$ value was determined using a two-sided log-rank test. Low CLDN1 mRNA expression was associated with significantly decreased overall survival ( $P=0.027)$. ( $E$ ) KaplanMeier estimates of overall survival in 67 patients with surgically resected lung adenocarcinoma: relationship to CLDN1 protein expression. CLDN1 protein expression was examined by immunohistochemical (IHC) analysis of primary tumor specimens from an independent cohort of patients with adenocarcinoma. Cases

were considered positive if more than $25 \%$ of the cancer cells showed membranous staining. Red line, patients with positive CLDN1 staining; blue line, patients with negative CLDN1 staining. $P$ value was determined using a two-sided log-rank test. Negative CLDN1 staining of tumor cells was associated with poor overall survival in patients with lung adenocarcinoma $(P=0.024)$.

Distinct patterns of claudin expression are found at the TJs in different organs and tissues. Altered claudin expression has been found in a wide variety of human malignancies, including colonic (8), ovarian (9), pancreatic (10), breast (11), liver (12), and prostate cancer (13).

Claudin (CLDN)-1, -3, -4, -5, and -7 expressions were detected in airway epithelial cells, whereas CLDN2, -6, -9, -10, -11, -15, and -16 were not detected in the lung (14). Lung cancer is by far the leading cause of cancer-related death in the world. The major obstacle to successful treatment is early metastasis, especially for non-small-cell lung carcinoma. Lung adenocarcinoma is the most common cell type of non-small-cell lung carcinoma. A recent study suggested that CLDN1 is downregulated in lung adenocarcinoma (15). We hypothesized that the TJ protein, CLDN1, may control the dissociation of cancer cells, and thereby play an important role in cancer cell invasion and metastasis. The aim of this study was to evaluate the clinical significance of CLDN1 expression in patients with lung adenocarcinoma and characterize the role of CLDN1 in cancer cell invasion and metastasis.

\section{METHODS}

\section{Patients and Tissue Sample Collections}

Lung adenocarcinoma tumor specimens for real-time quantitative reverse transcriptase-polymerase chain reaction (qRT-PCR) were obtained from 64 patients with a histologically confirmed diagnosis who underwent surgical resection at the Taichung Veterans General Hospital between November 22, 1999, and June 3, 2004. Immunohistochemical staining for CLDN1 was performed on tumor specimens from an independent cohort of 67 patients with lung adenocarcinoma who underwent surgical resection at the National Taiwan University Hospital from May 11, 1995, to December 14, 2000. None of the patients had received neoadjuvant chemotherapy or radiation therapy prior to surgery. The investigations were approved by the institutional review board of the Taichung Veterans General Hospital and National Taiwan University Hospital. The postsurgical pathologic stage of each tumor was classified according to the international tumor, node, and metastasis (TNM) classification (16).

\section{Antibodies}

Primary antibodies used for immunoblotting, immunofluorescence, and immunohistochemical staining were rabbit anti-CLDN1 (Zymed, San 
Francisco, CA), mouse anti-ZO-1 (Zymed, San Francisco, CA), and goat anti-connective tissue growth factor (CTGF) (Santa Cruz Biotechnology, Santa Cruz, CA).

\section{Video Time-lapse Microscopy}

Time-lapse images of migrating cells in serum-containing medium at $37^{\circ} \mathrm{C} / 5 \% \mathrm{CO}_{2}$ were collected on an inverted Zeiss Axiovert $200 \mathrm{M}$ microscope (Carl Zeiss, Jena, Germany) over the course of 12 hours. Images were obtained with a CoolSNAP HQ CCD camera (Roper Scientific, NJ) at 10-minute intervals and analyzed using MetaMorph 5.0 software (Universal Imaging, Downingtown, PA).

\section{Oligonucleotide Microarray Analysis}

The RNA preparation and array hybridization were performed according to the Affymetrix GeneChip Expression Analysis Technical Manual (Affymetrix, Santa Clara, CA) (47). Briefly, $8 \mu \mathrm{g}$ total RNA was reverse transcribed in the presence of a T7-(dT)24 primer using a One-cycle cDNA Synthesis kit (Affymetrix). The cDNA product was purified and transcribed in vitro with biotin-labeled ribonucleotides (IVT Labeling Kit; Affymetrix). A portion of the biotinylated RNA was fragmented and hybridized overnight to the Human Genome U133 plus 2.0 GeneChip (Affymetrix). The GeneChip was washed and developed according to the amplification staining protocol provided by Affymetrix. The GeneChip was scanned using an Affymetrix GeneChip Scanner 3000, and the images were extracted with Affymetrix GeneChip Operating Software, version 1.4. All hybridization experiments were performed in biological duplicate with cRNA probes prepared from two different CLDN1 transfectants (pc1513 and pc1515) and two different vector controls (p1511 and p1516). Data were filtered using a twofold change as a threshold, and the pathways associated with the resulting genes were analyzed using Genespring GX v7.3 (Silicon Genetics, Redwood City, CA).

\section{Statistical Analyses}

Data are presented as mean \pm SD. All statistical analyses were performed using SPSS for Windows, version 10.0 (SPSS Inc., Chicago, IL). The difference between two groups was analyzed using Student's $t$ test. Survival curves were obtained by the Kaplan-Meier method. Survival values were compared using the log-rank test. Multivariate Cox proportional hazards regression with stepwise selection was performed, with overall survival as the response variable. All statistical tests were two-sided, and a $P$ value less than 0.05 was considered statistically significant.

\section{RESULTS}

\section{CLDN1 Expression Predicts Clinical Outcome in Patients with Lung Adenocarcinoma}

In non-cancer patients the CLDN1 protein expression was detected in cell-cell junction of basal cells and columnar epithelium of the airways (Figure 1A). CLDN1 expression was also observed in lung cancer cells. High CLDN1 expression was detected in most squamous cell carcinomas (Figure 1B), as previously reported $(15,17)$. Conversely, loss of CLDN1 expression was not uncommonly seen in lung adenocarcinomas (Figure 1C). To explore whether CLDN1 expression is a prognostic factor in human lung adenocarcinoma, we examined the CLDN1 mRNA expression in 64 human lung adenocarcinomas by realtime quantitative RT-PCR. The relative amount of CLDN1 mRNA in tumor tissues was standardized against the geometric mean of TATA box binding protein (TBP) and glyceraldehyde 3phosphate dehydrogenase mRNAs, and individual samples were categorized as low or high CLDN1 expression using median expression level as a dividing line. Additional detail on the method for making these measurements is provided in the online supplement. Low CLDN1 mRNA expression was associated with significantly decreased overall survival (Figure 1D; $P=0.027$ ) in Kaplan-Meier log-rank analysis. Cox multivariate analysis
TABLE 1. CHARACTERISTICS OF 64 PATIENTS WITH LUNG ADENOCARCINOMA ANALYZED BY REAL-TIME QUANTITATIVE REVERSE TRANSCRIPTASE-POLYMERASE CHAIN REACTION

\begin{tabular}{lccc}
\hline & $\begin{array}{c}\text { High CLDN1 } \\
\text { Expression } \\
\text { Patient } \mathrm{n}(\%)\end{array}$ & $\begin{array}{c}\text { Low CLDN1 } \\
\text { Expression } \\
\text { Patient } \mathrm{n}(\%)\end{array}$ & P Value \\
\hline $\begin{array}{l}\text { Mean age } \pm \mathrm{SD}, \mathrm{yr} \\
\text { Sex }\end{array}$ & $66.5 \pm 11$ & $62.8 \pm 10.7$ & $0.183^{\star}$ \\
$\quad \begin{array}{l}\text { Male } \\
\text { Female }\end{array}$ & $23(72)$ & $22(69)$ & $1.0^{\dagger}$ \\
$\begin{array}{l}\text { Stage } \\
\text { I and II }\end{array}$ & $9(28)$ & $10(31)$ & \\
III & $16(50)$ & $14(47)$ & $0.805^{\dagger, \text { * }}$ \\
\hline
\end{tabular}

Definition of abbreviation: CLDN = claudin

* Student's $t$ test.

${ }^{\dagger}$ Fisher's exact test.

* Two patients' stage state were missing.

showed that the overall survival was associated with CLDN1 mRNA expression (low CLDN1 versus high CLDN1 hazard ratio [HR], 2.35; 95\% CI, 1.24-4.45; $P=0.009$ ) and stage (stage I and II versus stage III HR, 0.39; 95\% CI, 0.2-0.754; $P=0.005$ ).

CLDN1 protein expression was examined immunohistochemically in tumor specimens from an independent cohort of 67 patients with lung adenocarcinoma. Tumors in which more than $25 \%$ of the cancer cells showed membranous CLDN1 staining were considered positive, as previously described $(15,18)$. Representative images of CLDN1-positive and -negative lung adenocarcinoma identified by immunohistochemical staining are shown in Figure 1C. Negative CLDN1 staining of tumor cells was associated with poor overall survival in patients with lung adenocarcinoma (Figure 1E; $P=0.024$ ). Cox multivariate analysis also showed that the overall survival is associated with CLDN1 protein expression (negative CLDN1 versus positive CLDN1 HR, 1.9; 95\% CI, 1.08-3.34; $P=0.026$ ).

The clinical and pathologic features of the patient populations analyzed by real-time qRT-PCR and immunohistochemistry are summarized in Tables 1 and 2, respectively.

\section{CLDN1 Inhibits Cell Migration and Invasion In Vitro}

To determine whether CLDN1 has a causal role in tumor progression and invasion, we manipulated the expression levels of CLDN1 in the CL1-5 cell line. This cell line was chosen because it is a human lung adenocarcinoma cell line that has high invasion ability (19) and lacks detectable endogenous CLDN1 expression by Western blotting and real-time RT-PCR (data not shown). Human CLDN1 cDNA expression vectors or control vectors were transfected into the CL1-5 cells. After G418 selection, we isolated two clones (pc1513 and pc1515) with high CLDN1 expression, and

TABLE 2. CHARACTERISTICS OF THE INDEPENDENT COHORT OF 67 PATIENTS WITH LUNG ADENOCARCINOMA ANALYZED BY IMMUNOHISTOCHEMISTRY

\begin{tabular}{lccc}
\hline & $\begin{array}{c}\text { Positive CLDN1 } \\
\text { Staining } \\
\text { Patient n (\%) }\end{array}$ & $\begin{array}{c}\text { Negative CLDN1 } \\
\text { Staining } \\
\text { Patient n (\%) }\end{array}$ & P Value \\
\hline $\begin{array}{l}\text { Mean age } \pm \text { SD, yr } \\
\text { Sex }\end{array} \quad 63 \pm 9.9$ & $63 \pm 10.2$ & $0.997^{*}$ \\
$\quad$ Male & $11(33)$ & $19(56)$ & $0.087^{\dagger}$ \\
$\quad$ Female & $22(67)$ & $15(44)$ & \\
$\begin{array}{l}\text { Stage } \\
\text { I and II } \\
\text { III }\end{array}$ & $11(33)$ & $9(26)$ & $0.6^{\dagger}$ \\
\hline
\end{tabular}

For definition of abbreviation, see Table 1.

* Student's $t$ test.

${ }^{\dagger}$ Fisher's exact test. 

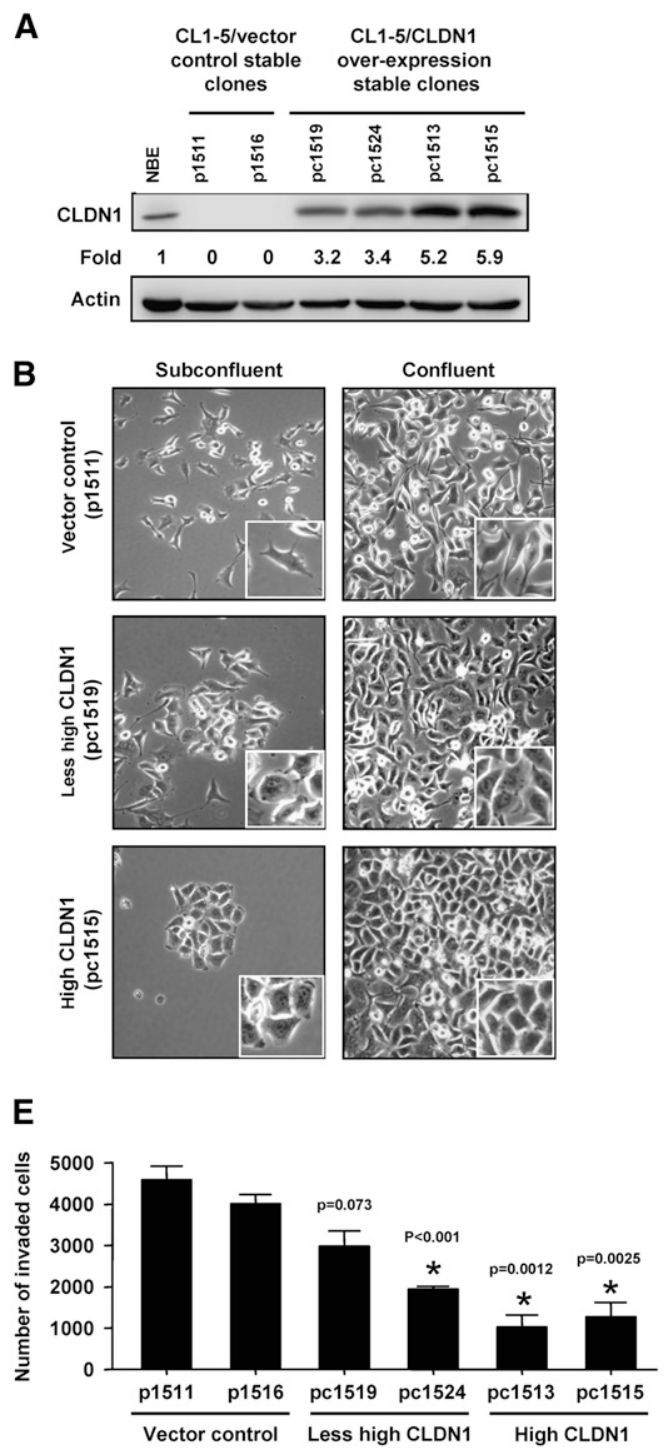

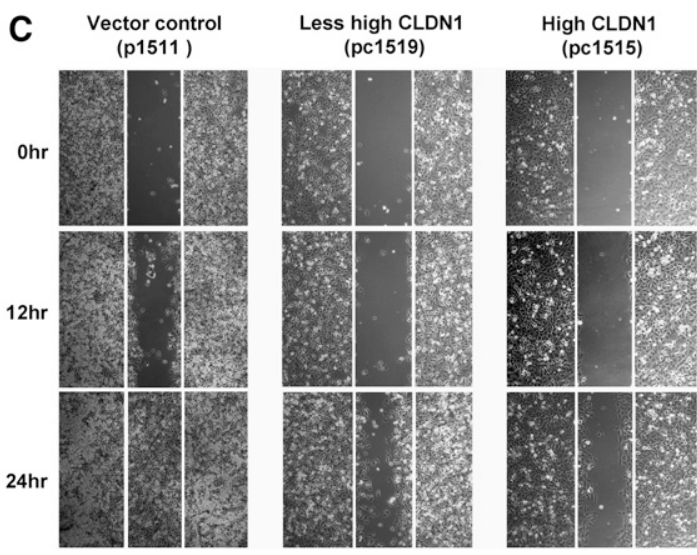

D
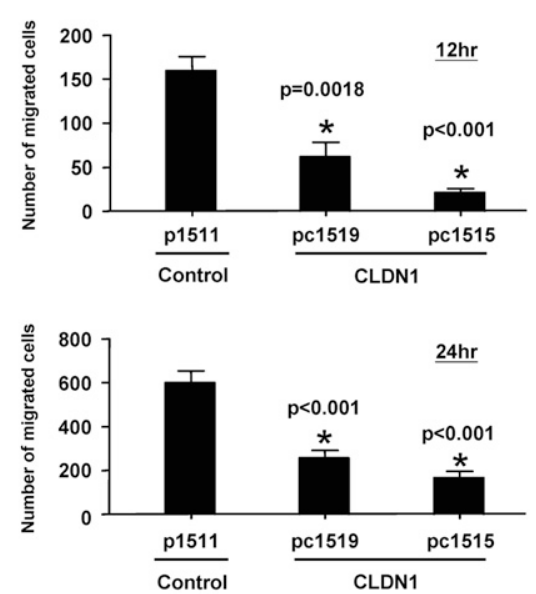

Figure 2. Expression of CLDN1 protein: effect on CL15 lung adenocarcinoma cell migration and invasion. ( $A$ ) Western blot analysis for the expression of CLDN1 in normal bronchial epithelium (NBE), selected stably transfected high CLDN1-expressing clones (pc1513 and pc1515), less high CLDN1-expressing clones (pc1519 and pc1524), and vector control clones (p1511 and p1516). Actin was used as a control for protein loading. Fold of CLDN1 expression was normalized to the expression of NBE. (B) Morphological appearance of subconfluent and confluent CL1-5/vector control (p1511) and CLDN1 overexpressing CL1-5/CLDN1 (pc1515, pc1519) cells, as shown by phase-contrast microscopy. The vector control cells exhibited a spindly, elongated, and dispersed morphology in monolayer culture like the parental CL1-5 cells, whereas the CLDN1-transfected cells exhibited an epithelial-like appearance, and tended to form multicellular aggregates. (C) CLDN1 expression and migration ability measured by scratch wound assay. Spontaneous motility of CL1-5/vector control (p1511) and CLDN1-overexpressing CL1-5/ CLDN1 (pc1515, pc1519) cells examined over a 24-hour period in confluent monolayers

wounded by a pipette tip. (D) Quantification of cells migrating into the wound area after 12 and 24 hours. Data are representative of three independent experiments and are expressed as mean \pm SD. ${ }^{*} P<0.05$ compared with vector control cells. The spontaneous motility displayed by $\mathrm{CL} 1-$ $5 / C L D N 1$ clones was greatly reduced compared with CL1-5/vector control. (E) CLDN1 expression and invasion ability. In vitro invasion activity of CL15/vector control (p1511 and p1516) and CLDN1-overexpressing CL1-5/CLDN1 (pc1513, pc1515, pc1519, and pc1524) cells was determined using a modified Boyden chamber by enumerating cells that migrated through Matrigel-coated filters (8- $\mu \mathrm{m}$ pore size) in Transwell chambers 24 hours after plating cells. Data are representative of three independent experiments and are presented as mean \pm SD. ${ }^{*} P<0.05$ compared with vector control p1516. There was a significant decrease in the invasive potential of the CL1-5/CLDN1 clones compared with CL1-5/vector control clones.

two clones (pc1519 and pc1524) that expressed CLDN1 at less elevated levels; two vector control clones (p1511 and p1516) were also isolated (Figure 2A). The vector control cells exhibited a spindly, elongated, and dispersed morphology in monolayer culture, like the parental CL1-5 cells, whereas the CLDN1-transfected cells exhibited an epithelial-like appearance and tended to form multicellular aggregates (Figure 2B). To assess the motility and invasive properties of these cell lines, we used scratch wound and modified Boyden chamber assays, respectively. In the scratch wound assay, the spontaneous motility displayed by CL1-5/ CLDN1 clones was greatly reduced compared with CL1-5/vector control clones, with high-CLDN1-expressing clones showing a greater reduction than less high-expressing clones (Figures 2C and 2D). In the modified Boyden chamber assay, there was a significant decrease in the invasive potential of the CL1-5/ CLDN1 clones compared with CL1-5/vector control clones (Figure 2E). The proliferation rates for the CL1-5/vector control cells and the CLDN1-overexpressing cells had also been checked, and showed no difference (data not shown). To further address the effect of CLDN1 expression on CL1-5 cell motility, we performed video time-lapse microscopy after wounding a confluent cell monolayer of green fluorescent protein (GFP)-CLDN1-transfected stable clone (GC-1550) and an enhanced GFP (EGFP)transfected stable clone (EGFP2) by scratching with a pipette tip. The EGFP2 cells rapidly migrated from the wounded edge into the wounded area. GC-1550 cells, however, were difficult to dissociate from each other and to move away from the wounded edge (Figure 3A; see also videos E1 and E2 for representative time-lapse microscopic images, available in the online supplement. Cell motility was analyzed by moving tracks and mean cell displacement of 20 individual cells over a 5-hour period. When compared with EGFP-transfected control, GFP-CLDN1-expressing cells showed dramatic compression of cell tracks (Figure 3B) and significantly decreased cell displacement (Figure 3C). These data indicate that overexpression of CLDN1 significantly suppressed the motility and invasive ability of CL1-5 cells in vitro. 
A
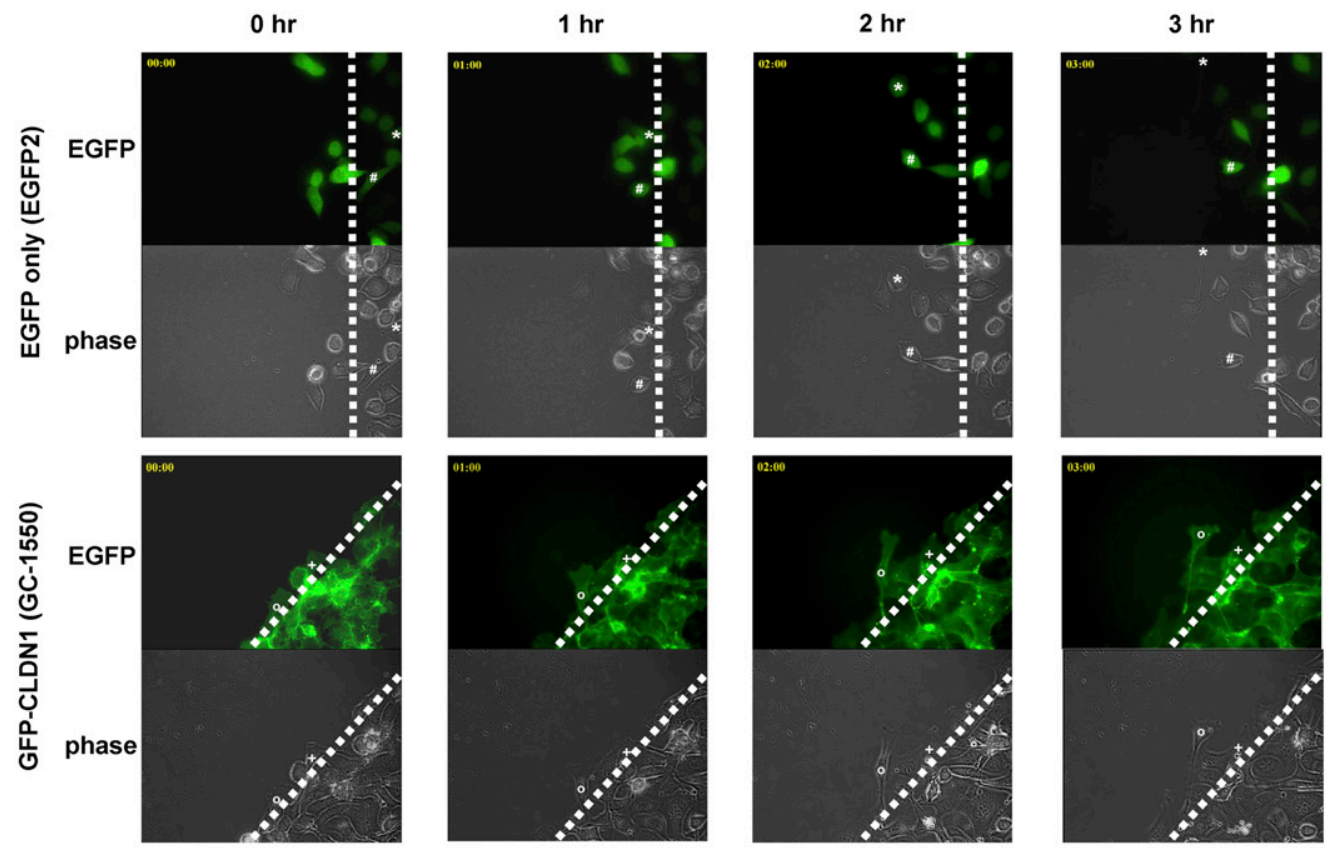

B

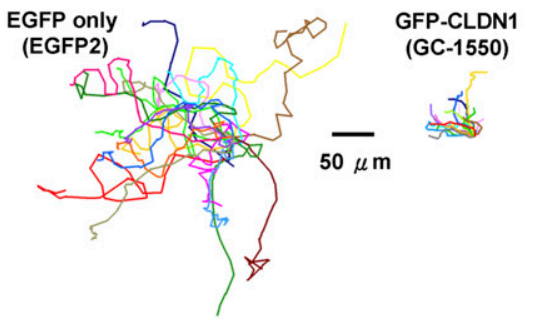

D
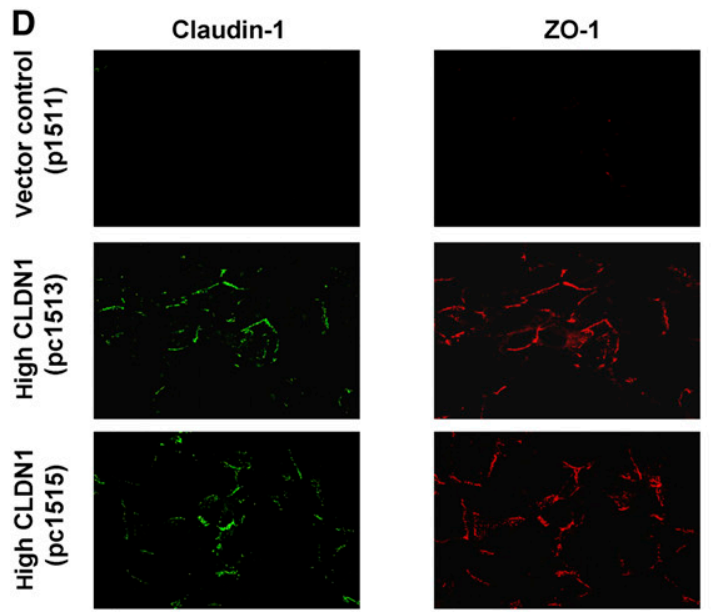

GPP-CLDN1

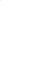
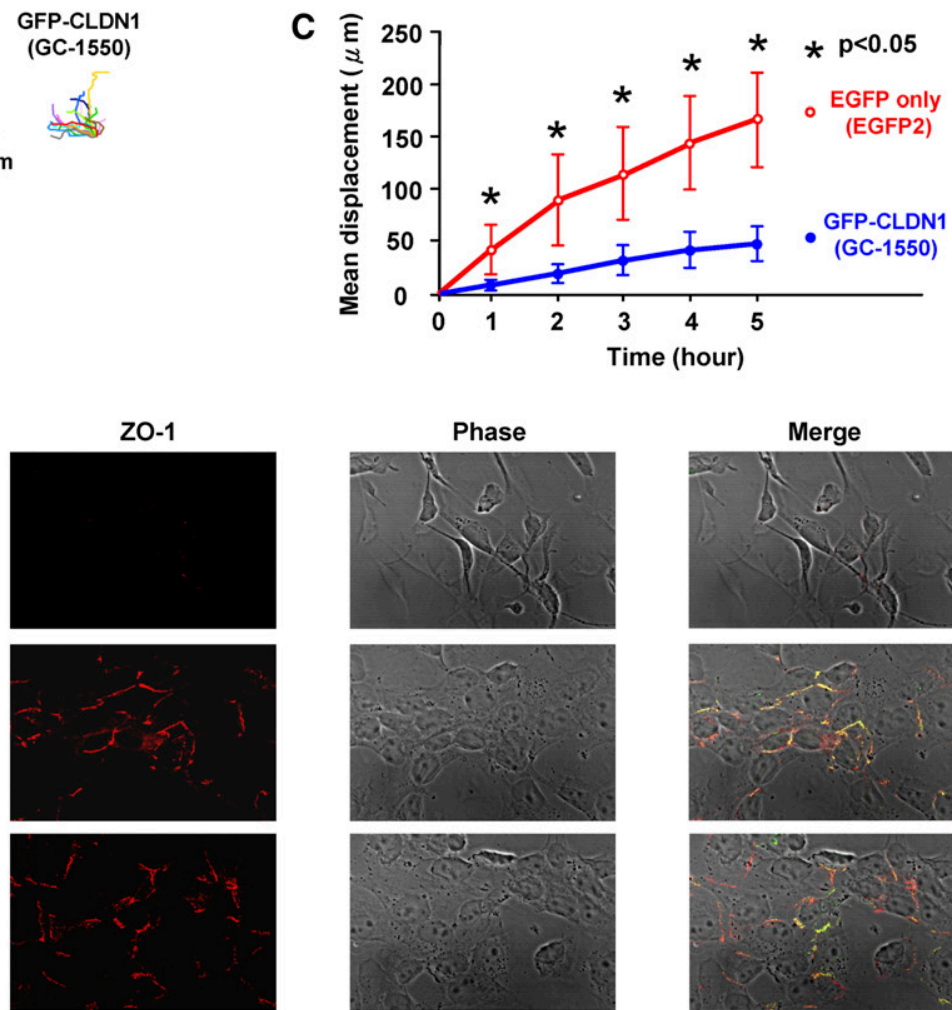
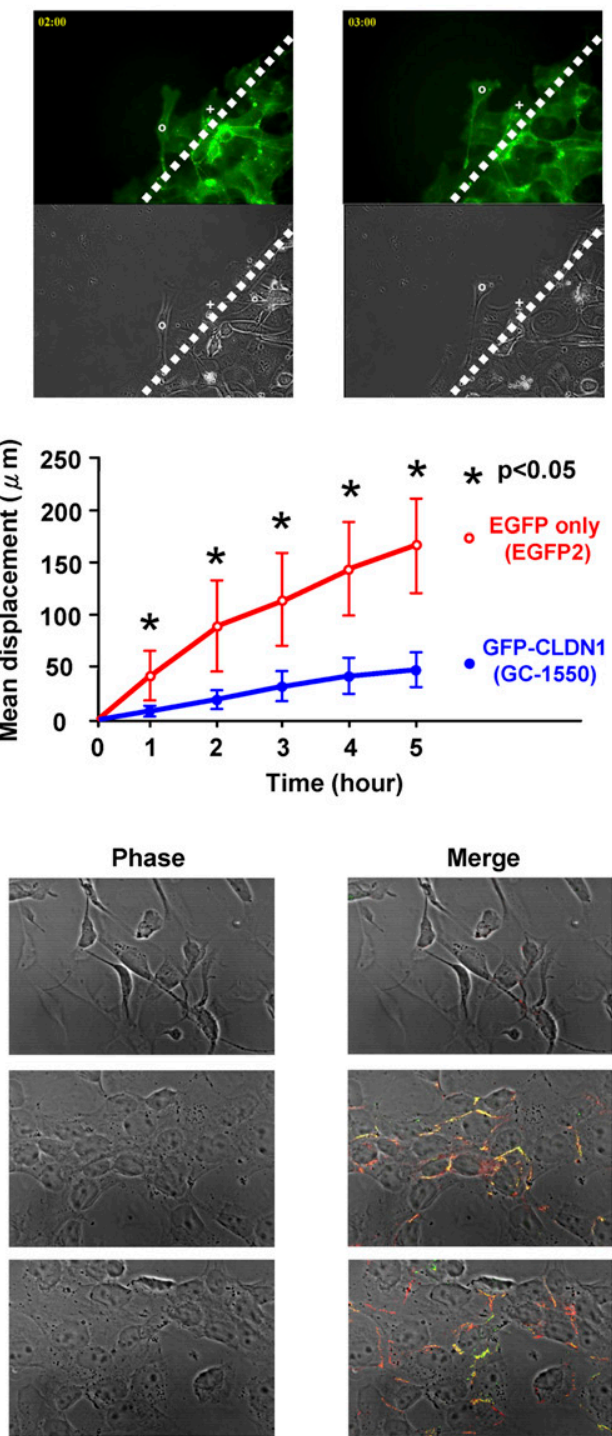
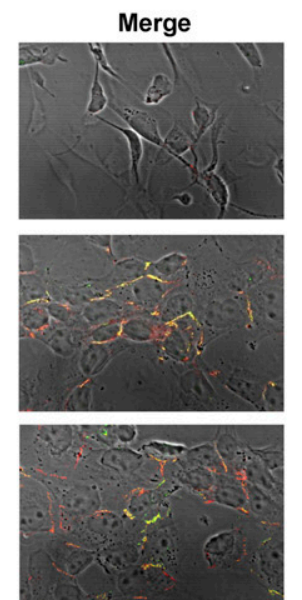

Figure 3. Time-lapse microscopic images of cell migration in GFP-labeled CLDN1-overexpressed cells and subcellular distribution of CLDN1 in CLDN1-transfected CL1-5 adenocarcinoma cells. (A) Representative time-lapse microscopic images of a GFP-CLDN1-transfected stable clone (GC-1550, CLDN1 overexpression) and an EGFP-transfected stable clone (EGFP2, control) after wounding a confluent cell monolayer with a pipette tip. Dashed line, wounded edge. $*, \#, 0,+$ Selected cells. The EGFP2 cells rapidly migrated from the wounded edge into the wounded area. However, the CLDN1-overexpressed cells were difficult to dissociate from each other and to move away from the wounded edge. For representative time-lapse images, see Videos E1 and E2 in the online supplement. (B) Analysis of cell motility of GFP-CLDN1-expressing cells (GC-1550) and an EGFP-transfected control (EGFP2). Moving tracks of 20 individual cells over a 5-hour period (different colors, normalized to their starting coordinates) showed the representative motility of cells. (C) Mean cell displacement from starting position of $(B)$. Red line, EGFP-transfected control (EGFP2); blue line, GFP-CLDN1expressing cells (GC-1550). Data are representative of 20 individual cells and are expressed as mean \pm SD. ${ }^{*} P<$ 0.05 compared with vector control cells at the same time point. GFP-CLDN1-expressing cells (GC-1550) showed significantly decreased cell displacement and dramatic compression of cell tracks. (D) Immunofluorescence localization of CLDN1 and ZO-1 in CLDN1-transfected (pc1513 and pc1515) and vector control (p1511) clones. Colocalization of CLDN1 and ZO-1 is indicated by yellow in the merged images. CLDN1 and ZO-1 staining were predominantly localized to the membrane of CL1-5/ CLDN1 cells at cell-cell contact points, a pattern that was morphologically similar to the tight junction (TJ) strand network in situ.

\section{Subcellular Distribution of CLDN1 in Adenocarcinoma Cells}

To determine the subcellular distribution of the CLDN1 in CL1-5/CLDN1 cells, the two high-CLDN1-expressing clones (pc1513 and pc1515) and one vector control clone (p1511) were stained with antibodies specific for CLDN1 and ZO-1 and analyzed by confocal immunofluorescence microscopy. As shown in Figure 3D, CLDN1 and ZO-1 staining were predominantly localized to the membrane of CL1-5/CLDN1 cells at cell-cell contact points, a pattern that was morphologically similar to the TJ strand network in situ. In contrast, the vector control p1511 cells showed no CLDN1 staining, and only low cytoplasmic intensity of ZO-1 staining.

\section{CLDN1 Inhibits Cancer Cell Metastasis}

Based on our cell culture data, we postulated that CLDN1 expression would affect cancer cell metastasis. To determine the impact of CLDN1 expression on metastatic colonization, we injected severe combined immunodeficiency (SCID) mice intravenously with the high-CLDN1-expressing clones, pc1513 and pc1515, or the vector control clone, p1511. At 6 weeks after 

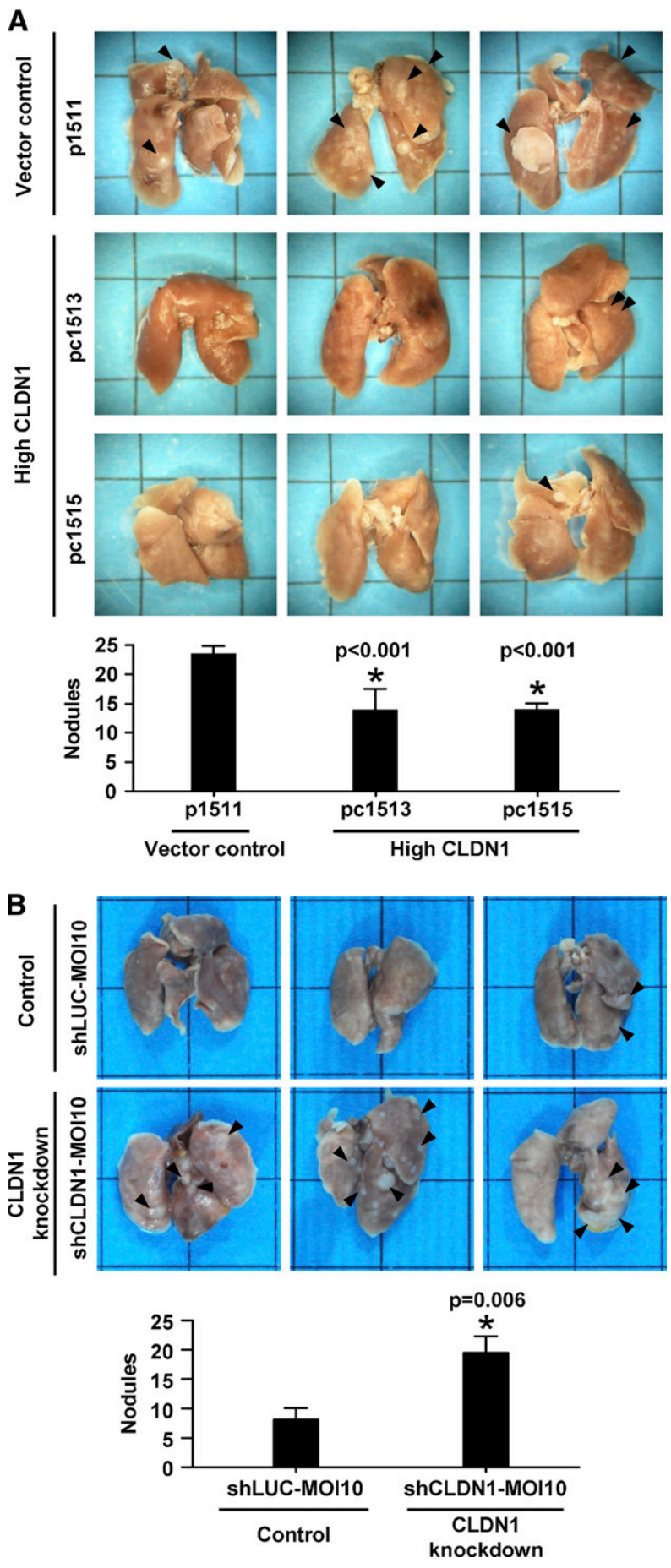

injection, all mice were killed and tumor formation in the lung was examined (Figure 4A, upper panel). Mice injected with CL1-5/vector control cells ( $\mathrm{p} 1511$ ) had numerous lung metastases, whereas mice injected with CLDN1-overexpressing cells (pc1513 and pc1515) had fewer detectable tumor nodules in the lungs (Figure 4A, lower panel). Thus, overexpression of CLDN1 in CL1-5 cells suppressed the ability of these cells to form metastatic nodules in the lungs.

\section{Knockdown of Exogenous CLDN1 Expression Restores Cancer Cell Invasive and Metastatic Abilities}

To further confirm the association of CLDN1 expression and cancer cell invasive and metastatic capability, we used a CLDN1
Figure 4. Suppression of $\mathrm{CL} 1-5$ lung adenocarcinoma cell metastasis by CLDN1. (A) $10^{6}$ cells were suspended in $0.1 \mathrm{ml}$ phosphate-buffered saline (PBS) and injected into the lateral tail vein of SCID mice (10 mice/group). At 6 weeks after injection, all mice were killed and the number of lung surface tumor foci was determined. Upper panel: representative lungs from mice injected intravenously with high CLDN1-expressing (pc1513 and pc1515) or vector control (p1511) clones. Arrowheads: metastatic tumor nodules. Lower panel: quantitative evaluation of lung metastatic nodules 6 weeks after inoculation. Data are expressed as mean $\pm S D$. ${ }^{*} P<0.05$. Mice injected with $\mathrm{CL} 1-$ $5 /$ vector control cells had numerous lung metastases, whereas mice injected with CLDN1 overexpressing cells had fewer detectable tumor nodules in the lungs. (B) Upper panel: representative lungs of mice injected intravenously with short hairpin (sh) CLDN1 knockdown (shCLDN1-multiplicity of infection [MOI]-10) or negative control (shLUC-MOI10) cells. Arrowheads: metastatic tumor nodules. Lower panel: Quantitative evaluation of lung metastatic nodules 6 weeks after inoculation. Data are expressed as mean \pm SD. ${ }^{*} P<0.05$. Mice injected with shCLDN1 knockdown cells developed significantly more pulmonary metastatic nodules than did those injected with negative control cells.

targeting short hairpin (sh) RNA lentivirus (shCLDN1) to inhibit exogenous CLDN1 expression in CL1-5/CLDN1 cells (pc1515). As a negative control, pc1515 cells were infected with a luciferase shRNA lentivirus (shLUC). Infection of pc1515 cells with shCLDN1 using different virus titers (multiplicity of infection $[\mathrm{MOI}]=1,4$, or 10 ) induced a significant, dosedependent reduction of exogenous CLDN1 expression (Figure 5A). shCLDN1 knockdown cells exhibited a spindly, elongated, and dispersed morphology in monolayer culture, whereas the negative control cells retained an epithelial-like appearance that was similar to pc1515 cells (Figure 5B). The spontaneous motility of shCLDN1 knockdown cells was increased relative to negative control cells (Figures 5C and 5D). Furthermore, the invasive activity was increased approximately twofold after knockdown of exogenous CLDN1 expression in pc1515 cells (Figure 5E). Mice injected with shCLDN1 knockdown cells (shCLDN1-MOI10) developed significantly more pulmonary metastatic nodules than did those injected with negative control cells (shLUC-MOI10) (Figure 4B).

\section{Knockdown of Endogenous CLDN1 Expression Increases the Invasive Ability of HOP62 Lung Adenocarcinoma Cells}

To ascertain whether reduced endogenous CLDN1 expression can increase lung adenocarcinoma cancer cell metastasis, we selected another lung adenocarcinoma cell line with relatively lower invasive ability: HOP62. We knocked down the endogenous CLDN1 in HOP62 cells by the shRNA approach (Figure $6 \mathrm{~A})$, and found that the negative control cells exhibited epithelial morphology and formed multicellular aggregates like the parental HOP62 cells. However, the CLDN1-knockdown cells exhibited spindly, elongated, and dispersed mesenchymal morphology (Figure 6B). In the modified Boyden chamber invasion assay, there was a significant increase in the invasive potential of the CLDN1-knockdown cells compared with negative control cells (Figure 6C).

\section{Gene Expression Changes Associated with CLDN1 Overexpression}

To identify potential genes altered by CLDN1 expression, we analyzed CL1-5/CLDN1 transfectants (pc1513 and pc1515) and vector control clones (p1511 and p1516) for differential gene expression using Affymetrix oligonucleotide microarrays. A 


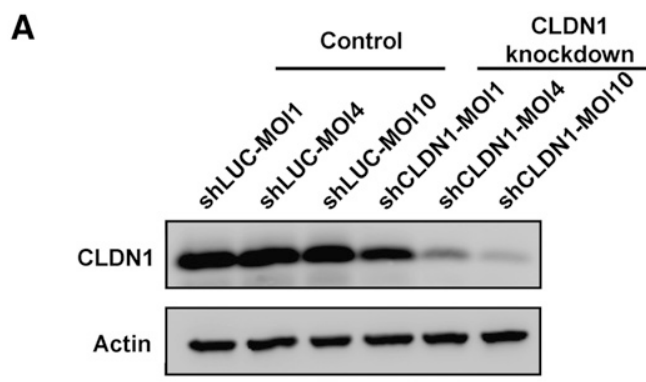

B
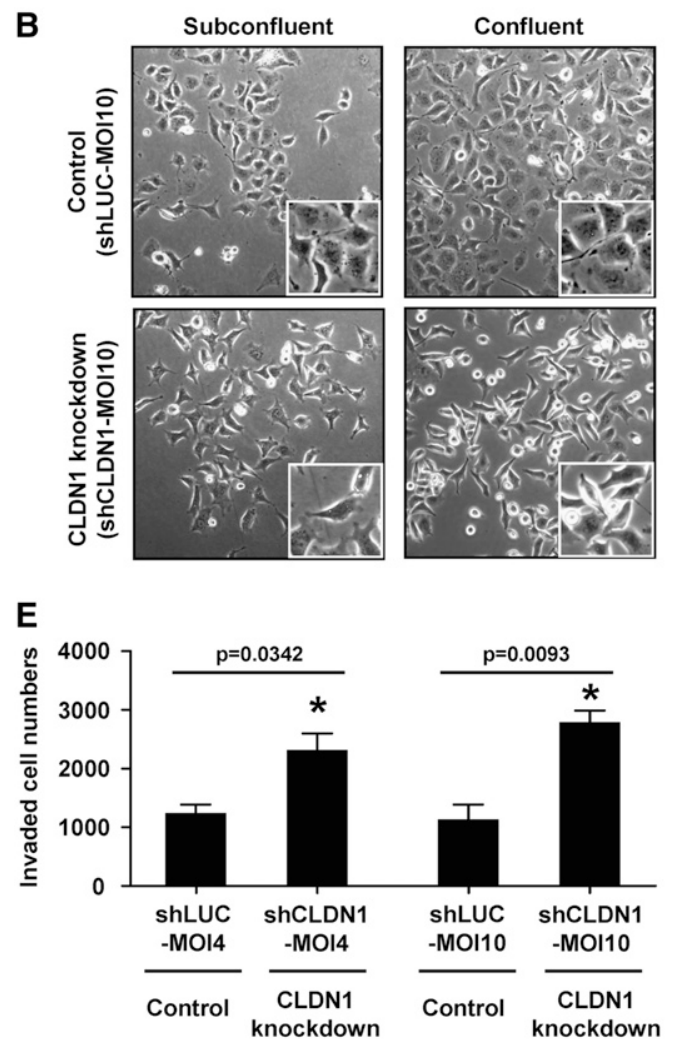

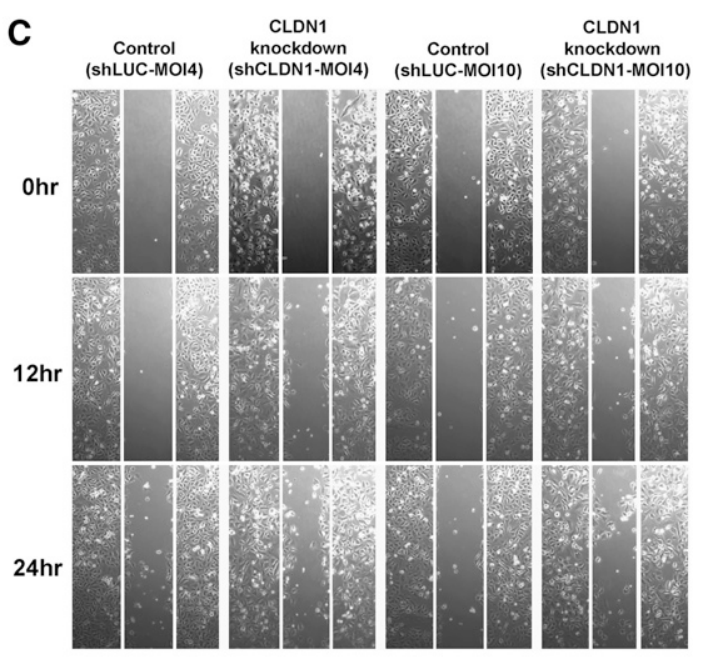

D
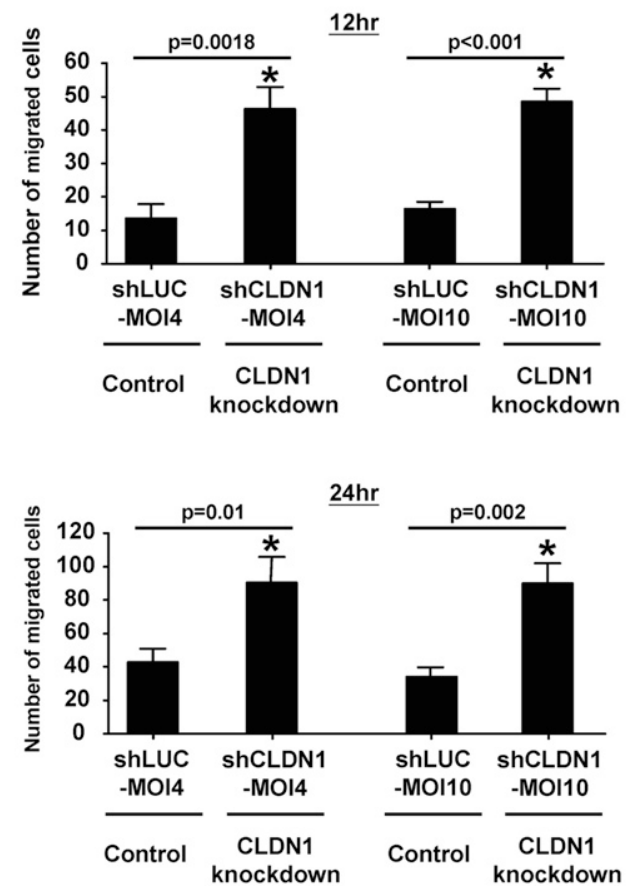

Figure 5. Knockdown of exogenous CLDN1 expression in CL1-5/CLDN1 cells restores the migratory and invasive ability. (A) Western blot analysis of CLDN1 expression in selected knockdown clones (pooled) generated by infecting pc 1515 with CLDN1-targeting shRNA lentivirus (shCLDN1) using different virus titers $(\mathrm{MOI}=1,4$, or 10). Luciferase shRNA lentivirusinfected pc1515 cells (shLUCMOI1, -MOI4, -MOI10) served as negative controls, and actin was used as a control for protein loading. (B) Morphological appearance of subconfluent and confluent CLDN1 knockdown shCLDN1-MOI10 cells and shLUC-MOI10 cells, as shown by phase-contrast microscopy. shCLDN1 knockdown cells exhibited spindly, elongated, and dispersed morphology in monolayer culture, whereas the negative control cells retained an epithelial-like appearance that was similar to pc1515 cells. (C) The migratory ability of CLDN1 knockdown shCLDN1 cells measured by scratch wound assay. Left two columns, shLUC-MOI4 and shCLDN1-MOI4 cells; right two columns, shLUC-MOI10 and shCLDN1-MOI10 cells. (D) Upper panel: quantification of cells migrating into the wound area after 12 hours. Lower panel: quantification of cells migrating into the wound area after 24 hours. Data are representative of three independent experiments and are expressed as mean \pm SD. ${ }^{*} P<0.05$ com-

pared with negative control cells. The spontaneous motility of shCLDN1 knockdown cells was increased relative to negative control cells. ( $E$ ) In vitro invasion activity of shCLDN1 knockdown cells (shCLDN1-MOI4, -MOI10) and negative control (shLUC-MOI4, -MOI10) cells was determined using a modified Boyden chamber by enumerating cells that migrated through Matrigel-coated filters ( $8-\mu \mathrm{m}$ pore size) in Transwell chambers 24 hours after plating. Data are representative of three independent experiments and are expressed as mean \pm SD. ${ }^{*} P<0.05$ compared with negative control cells. The invasive activity was increased approximately twofold after knockdown of exogenous CLDN1 expression in pc1515 cells.

total of 773 genes exhibited at least a two-fold change in expression levels between CL1-5/CLDN1 and CL1-5/vector control (see Table E1 in the online supplement). A number of genes associated with cancer invasion or metastasis were increased or suppressed by CLDN1 overexpression. The original data obtained from Affymetrix human genome U133 plus 2.0 GeneChip have been deposited in the National Center for Biotechnology Information database (GSE10309: http://www.ncbi.nlm.nih. gov/geo/query/acc.cgi?token $=$ xvilrqimuymsqtiandacc $=$ GSE10309; http://genestamp.ibms.sinica.edu.tw/cldn1/index.html). The potential functional implications of the identified genetic changes were analyzed using Genespring software, which indicated that several signaling pathways and cellular components or processes were influenced, including those involving ECM-receptor interaction, cell communication, TGF- $\beta$ signaling, TJs, complement and co- agulation cascades, focal adhesion, apoptosis, and actin cytoskeletal regulation. Several genes representing different cellular functions, including a transcriptional regulator, a GTPase, an extracellular matrix protein, a transporter, a growth factor, and constituents of TJs, were selected to validate the microarray result using semiquantitative RT-PCR (Figure 7A). We also chose ZO-1 and CTGF to verify changes at the protein level. ZO-1 and CTGF proteins were increased in cells stably expressing CLDN1 transfectants, but decreased after exogenous CLDN1 expression was knocked down (Figure 7B).

CLDN1 is known to modulate matrix metalloproteinase (MMP) activity (20). To determine whether MMPs are activated by CLDN1, we used gelatin zymography to assess the enzyme activity of MMPs in CL1-5/CLDN1 and CL1-5/vector control cells. The results revealed that overexpression of 
A
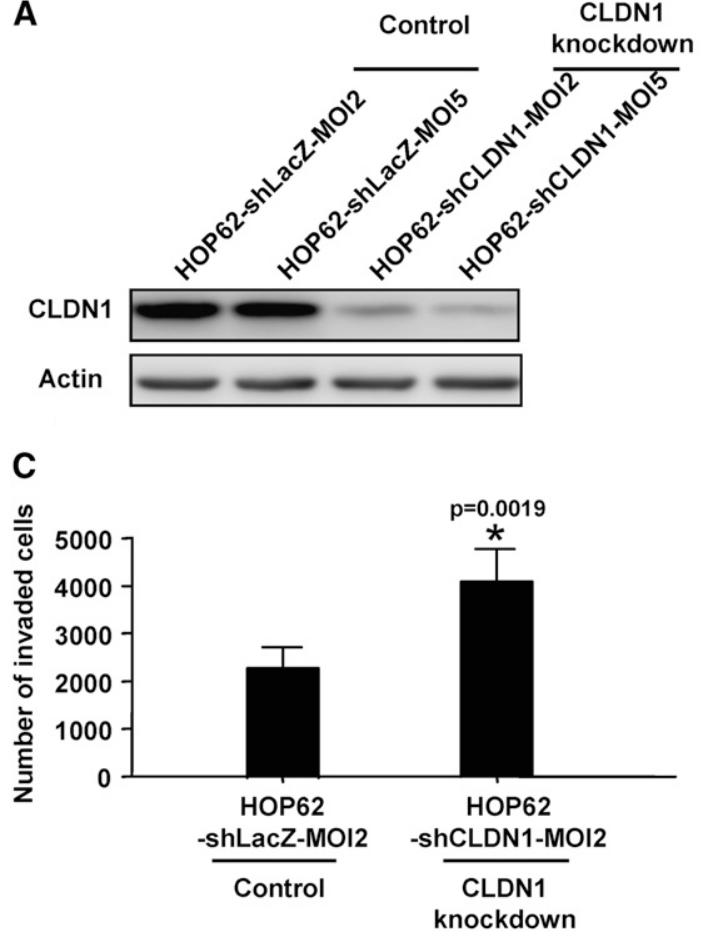

B
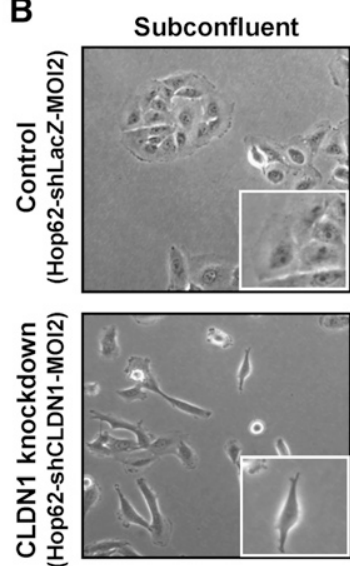

2.
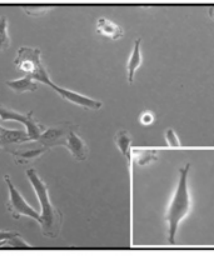
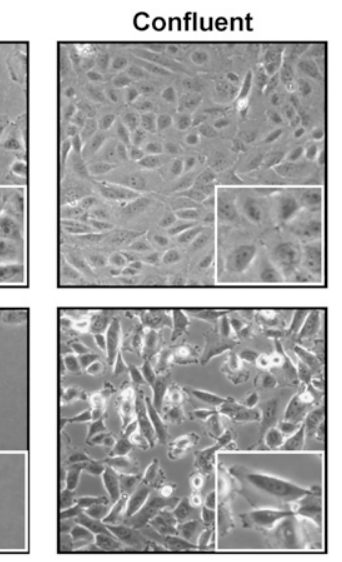

Figure 6. Knockdown of endogenous CLDN1 expression in HOP62 lung adenocarcinoma cells: effect on cell morphology and invasion. (A) Western blot analysis of CLDN1 expression in selected knockdown clones (pooled) generated by infecting HOP62 cells with CLDN1targeting shRNA lentivirus (shCLDN1) using different virus titers $(\mathrm{MOI}=2$, or 5$)$. LacZ shRNA lentivirus-infected HOP62 cells (shLacz-MOI2, -MOI5) served as negative controls, and actin was used as a control for protein loading. (B) Morphological appearance of subconfluent and confluent CLDN1 knockdown HOP62shCLDN1-MOI2 cells and HOP62-shLacZ-MOI2 cells, as shown by phase-contrast microscopy. shCLDN1 knockdown cells exhibited a spindly, elongated, and dispersed mesenchymal morphology in monolayer culture, whereas the negative control cells retained an epithelial-like appearance that was similar to parental HOP62 cells. (C) In vitro invasion activity of shCLDN1 knockdown cells (HOP62-shCLDN1-MOI2) and negative control (HOP62-shLacZ-MOI2) cells was determined using a modified Boyden chamber by enumerating cells that migrated through Matrigel-coated filters (8- $\mu \mathrm{m}$ pore size) in Transwell chambers 24 hours after plating. Data are representative of three independent experiments and are expressed as mean \pm SD. ${ }^{*} p<0.05$ compared with negative control cells. The invasive activity was increased approximately twofold after knockdown of endogenous CLDN1 expression in HOP62 cells.

CLDN1 significantly increased the activity of MMP2, while that of MMP9 was unaltered (Figure 7C).

\section{DISCUSSION}

The results of this study show that CLDN1 is a cancer invasion and metastasis suppressor. Patients with lung adenocarcinoma with low CLDN1 expression had shorter overall survival. Overexpression of CLDN1 inhibited lung adenocarcinoma cells from dissociating from each other in time-lapse imaging of wound healing, and suppressed cell migration, invasion, and metastatic activity. Knockdown of exogenous or endogenous CLDN1 expression increased invasivity and metastatic ability. A microarray analysis identified several modulators of cancer invasion or metastasis that were altered by CLDN1 overexpression, including CTGF, THBS1, DLC1, OCLN, ZO-1, SPP1, CUTL1, TGF- $\alpha$, SLC2A3, and PGF, lending further support to the conclusion that CLDN1 functions to suppress cancer invasion and metastasis.

Disruption of the cell-cell junction and detachment of tumor cells from the primary site is the first step of cancer cell invasion and metastasis. Our data show that CLDN1 overexpression induced formation of TJ-like strands on the membrane at cellcell contacts (Figure 3D), and transformed cells from a fibroblastoid morphology to an epithelial-like cell that tended to form multicellular aggregates (Figure 2B). They also exhibited reduced motility and invasivity (Figures $2 \mathrm{C}$ and $2 \mathrm{E}$ ). In addition, we found that CLDN1 transfectants were dissociation resistant and difficult to move away from the wounded edge of cell monolayers (Figure 3A). Because cell dissociation, motility, and invasivity are key early events in the metastatic process, these data suggest that CLDN1 may inhibit cancer progression at the initial step of metastasis.

We also found that CLDN1 has prognostic value in patients with lung adenocarcinoma, showing that low CLDN1 mRNA expression and negative CLDN1 staining of tumor cells was associated with significantly decreased overall survival. Various types of cancer have been shown to exhibit altered CLDN1 expression, which, in most studies, has been shown to have prognostic significance. For example, decreased expression of CLDN1 was recently reported to correlate with the recurrence status and malignant potential of breast cancer (21). Loss of CLDN1 was also found to play a significant role in the acquisition of the metastatic phenotype in cutaneous melanoma (22), hepatocellular carcinoma (23), and prostate cancer (24).

Many of the CLDN1 altered genes identified by Affymetrix microarray have previously been described as modulators of cancer invasion or metastasis, and have also frequently been found to have prognostic value. For example, one of the increased genes, CTGF, is a member of the (CYR61/CTGF/ NOV) (CCN) family that encodes a secreted growth factor capable of binding to integrins on the cell surface. We previously reported that CTGF induced the expression of the metastasis suppressor, collapsin response mediator protein 1 (CRMP-1) (25), via a pathway mediated by the integrins, $\alpha_{\mathrm{v}} \beta_{3}$ and $\alpha_{v} \beta_{5}(26)$. CTGF also modulates invasive behavior, and acts as an independent prognostic marker in lung adenocarcinoma (26). Other genes found to be increased in our screen include THBS1, which is a multifunctional extracellular matrix protein that suppresses tumorigenesis, angiogenesis, and tumor metastasis in breast cancer (27), and DLC-1, which encodes a Rho GTPase-activating protein that functions as a metastasis suppressor gene in non-small-cell lung cancer (28) and breast 


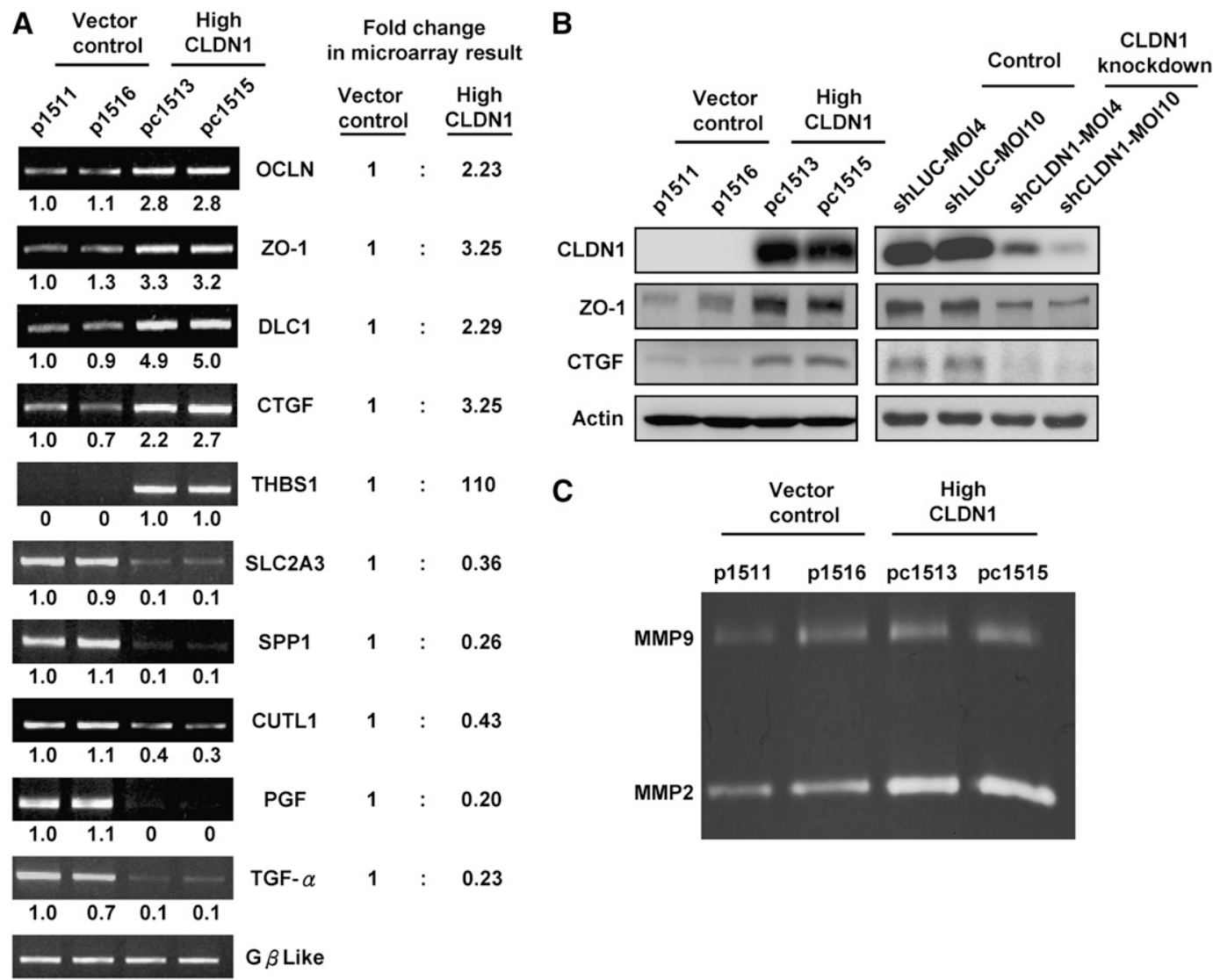

Figure 7. Validation of the microarray result and gelatinase activity of matrix metalloproteinase (MMPs) in CL1-5/CLDN1 and $\mathrm{CL} 1-5 /$ vector control cells. (A) Correlation between microarray and semiquantitative RTPCR results for selected CLDN1 target genes. RNA from stable CL1-5/CLDN1 and CL1-5/vector control clones was isolated, reverse transcribed, amplified using primers specific for each gene (listed in Table E2) and resolved on agarose gels (shown at left). Corresponding relative expression values, as determined by microarray analysis, are shown in the right-hand column. (B) Western blot analysis of CLDN1, ZO-1, and connective tissue growth factor (CTGF) expression in CL1-5/CLDN1 (pc1513 and pc1515), CL1-5/ vector (p1511 and p1516), and CLDN1 knockdown clones (pooled) generated by infecting pc1515 with CLDN1-targeting shRNA lentivirus (shCLDN1) using different virus titers (MOI4, MOI10). Luciferase shRNA lentivirus (shLUC)-infected pc1515 cells (shLUC-MOI4, -MOI10) served as negative controls, and actin was used as a control for protein loading. ZO-1 and CTGF proteins were increased in cells stably expressing CLDN1 transfectants, but decreased after exogenous CLDN1 expression was knocked down. (C) Activities of MMP-2 and MMP-9 in CL1-5/ CLDN1 (pc1513 and pc1515) and CL1-5/vector (p1511 and p1516) cells determined by gelatin zymography. The results revealed that overexpression of CLDN1 significantly increased the activity of MMP2, while that of MMP9 was unaltered.

cancer cells (29). Also in the increased group were ZO-1 and OCLN, which are integral constituents of TJs that are reduced during tumor formation and metastasis $(4,5,30)$. Among the suppressed genes are included SLC2A3, which is a transmembrane protein with a high affinity for glucose that facilitates glucose transport, and SPP1, which encodes a multifunctional $34 \mathrm{kD}$ extracellular matrix protein. Elevated expression of SLC2A3 (31) or SPP1 (32) in stage I non-small-cell lung carcinoma has been associated with poor survival. CUTL1, which was also suppressed, is a homeodomain transcriptional regulator that is associated with increased migration and invasiveness in breast cancer and is highly expressed in high-grade breast cancers (33). Other suppressed genes include PGF, a member of the vascular endothelial growth factor family of proangiogenic factors, and TGF- $\alpha$, a well characterized growth factor that interacts with the epidermal growth factor receptor and activates its intrinsic tyrosine kinase. PGF expression levels are significantly correlated with serosal invasion, positive lymph node metastases, tumor stages, and patient survival in gastric cancer (34), and TGF- $\alpha$ has been implicated in the metastatic spread of colon cancer cells (35).

There are some reports that CLDN1 expression enhances invasive ability and metastatic properties, possibly by increasing MMP-2 activity. This has been shown, for example, in colon carcinoma (36), melanoma (37), and oral squamous cell carcinoma (38). CLDN1 has been shown to directly interact with membrane-type matrix metalloproteinase-1 (MT1-MMP) and pro-MMP-2, recruiting them to the cell surfaces, where it consequently activates pro-MMP-2 (20). Interestingly, we found that the activity of MMP-2 was also increased in our CLDN1tranfected clones (Figure 7C), but, instead of increasing invasivity and metastatic potential, CLDN1 decreased both of these properties (Figures 2D and 4A). One possible explanation for these conflicting results lies in the fact that the claudin family consists of at least 24 members; in different organs and different tissues, the patterns of claudin expression at TJs are distinctly different. These differences have functional consequences, accounting for the observed differences in permeability and electrical resistance of various epithelia (39). Thus, claudin expression patterns may vary along with the histology of the tumor. For example, CLDN5 expression was detected in adenocarcinomas, but not in squamous cell carcinomas. High CLDN1 expression was detected in most squamous cell carcinomas (Figure 1B) $(15,17)$. Conversely, loss of CLDN1 expression was not uncommonly seen in lung adenocarcinomas (Figure 1C) (15). CLDN1 immunoreactivity was not detected in normal colon epithelial cells, but cells from primary colon adenocarcinoma, and also those from metastatic lesions, stained intensely for CLDN1 $(8,36)$. In contrast, significant levels of CLDN1 immunoreactivity were found in normal bronchial cells (Figure 1A). It is possible that the effect of introducing exogenous CLDN1 into the colonic epithelium may be different from restoring endogenous CLDN1 expression in the lung epithelium. Thus, the apparent discrepancies in the role of CLDN1 in cancer progression may reflect the specific contribution of different cellular contexts. Resolving this issue will 
require a better understanding of the specific roles of different claudins and their potential to interact with specific cellular environments and function in different types of tumor cells.

The TNM staging system is used to determine the anatomical extent of malignant disease, which provides prognostic information and guides appropriate therapy. In the TNM system, the presence or absence of distal metastases is more difficult to evaluate rigorously than the tumor size or nodal spread, because occult microscopic metastases can go undetected in a staging evaluation (40). Besides, the TNM system may be misleading when applied to small tumors with high invasivity, which causes early hematogenous dissemination, or large tumors but with low invasivity. In breast cancer, the TNM staging correlates broadly with prognosis, but more reliable indices of prognosis can be calculated from several clinicopathological variables and biological markers (41). Gene expression profiles from primary lung adenocarcinoma have been described to predict lymph node metastasis and help to detect occult disease spread (42). There are several metastasis-related genes that are uncorrelated with stages, such as RhoGDI2 (43), BRMS1 (44), HLJ1 (45), and NRP1 (46). As a tumor metastasis suppressor gene, CLDN1 may assist to predict tumor invasive potential and occult microscopic metastatic disease in lung adenocarcinoma. Thus, it is reasonable to find that CLDN1 expression is not correlated with stages (Tables 1 and 2), but with survival of patients.

Taken together, these data strongly suggest that CLDN1 is a cancer invasion/metastasis suppressor. Overexpression of CLDN1 in cancer cells not only promotes MMP-2 activation, but also influences the expression of several important tumor invasiveness- and metastasis-related genes. Additional mechanisms and downstream signaling pathways relevant to CLDN1 expression in lung adenocarcinoma need further investigation. However, the present observations raise the possibility that CLDN1 could be exploited as a biomarker for lung adenocarcinoma progression, and might provide new opportunities for therapeutic intervention.

Conflict of Interest Statement: None of the authors has a financial relationship with a commercial entity that has an interest in the subject of this manuscript.

Acknowledgments: shRNA constructs were obtained from the National RNAi Core Facility located at the Institute of Molecular Biology/Genomic Research Center, Academia Sinica, supported by National Research Program for Genomic Medicine grants of NSC (NSC-94-3112-B-001-003 and NSC-94-3112-B-001018-Y). The authors thank National Taiwan University Center of Genomic Medicine and Microarray Core Facility for microarray analysis and technical support. T.-M.H. and P.-C.Y. codirected the project and contributed equally to this work.

\section{References}

1. Braga VM. Cell-cell adhesion and signalling. Curr Opin Cell Biol 2002; 14:546-556.

2. Wittekind C, Neid M. Cancer invasion and metastasis. Oncology 2005; 69:14-16.

3. Sawada N, Murata M, Kikuchi K, Osanai M, Tobioka H, Kojima T, Chiba $\mathrm{H}$. Tight junctions and human diseases. Med Electron Microsc 2003;36:147-156.

4. Hoover KB, Liao SY, Bryant PJ. Loss of the tight junction maguk ZO-1 in breast cancer: relationship to glandular differentiation and loss of heterozygosity. Am J Pathol 1998;153:1767-1773.

5. Gu JM, Lim SO, Park YM, Jung G. A novel splice variant of occludin deleted in exon 9 and its role in cell apoptosis and invasion. FEBS $J$ 2008;275:3145-3156.

6. Tsukita S, Furuse M, Itoh M. Multifunctional strands in tight junctions. Nat Rev Mol Cell Biol 2001;2:285-293.

7. Morita K, Furuse M, Fujimoto K, Tsukita S. Claudin multigene family encoding four-transmembrane domain protein components of tight junction strands. Proc Natl Acad Sci USA 1999;96:511-516.

8. Miwa N, Furuse M, Tsukita S, Niikawa N, Nakamura Y, Furukawa Y. Involvement of claudin- 1 in the $\beta$-catenin/TCF signaling pathway and its frequent upregulation in human colorectal cancers. Oncol Res 2001;12:469-476.

9. Rangel LB, Agarwal R, D'Souza T, Pizer ES, Alo PL, Lancaster WD, Gregoire L, Schwartz DR, Cho KR, Morin PJ. Tight junction proteins claudin-3 and claudin-4 are frequently overexpressed in ovarian cancer but not in ovarian cystadenomas. Clin Cancer Res 2003:9:2567-2575.

10. Michl P, Barth C, Buchholz M, Lerch MM, Rolke M, Holzmann KH, Menke A, Fensterer H, Giehl K, Lohr M, et al. Claudin-4 expression decreases invasiveness and metastatic potential of pancreatic cancer. Cancer Res 2003;63:6265-6271.

11. Kominsky SL, Argani P, Korz D, Evron E, Raman V, Garrett E, Rein A, Sauter G, Kallioniemi OP, Sukumar S. Loss of the tight junction protein claudin-7 correlates with histological grade in both ductal carcinoma in situ and invasive ductal carcinoma of the breast. Oncogene 2003;22:2021-2033.

12. Cheung ST, Leung KL, Ip YC, Chen X, Fong DY, Ng IO, Fan ST, So S. Claudin-10 expression level is associated with recurrence of primary hepatocellular carcinoma. Clin Cancer Res 2005;11:551-556.

13. Long H, Crean CD, Lee WH, Cummings OW, Gabig TG. Expression of clostridium perfringens enterotoxin receptors claudin-3 and claudin- 4 in prostate cancer epithelium. Cancer Res 2001;61:7878-7881.

14. Coyne CB, Gambling TM, Boucher RC, Carson JL, Johnson LG. Role of claudin interactions in airway tight junctional permeability. Am J Physiol 2003;285:L1166-L1178.

15. Paschoud S, Bongiovanni M, Pache JC, Citi S. Claudin-1 and claudin-5 expression patterns differentiate lung squamous cell carcinomas from adenocarcinomas. Mod Pathol 2007;20:947-954.

16. Mountain CF. Revisions in the international system for staging lung cancer. Chest 1997;111:1710-1717.

17. Moldvay J, Jackel M, Paska C, Soltesz I, Schaff Z, Kiss A. Distinct claudin expression profile in histologic subtypes of lung cancer. Lung Cancer 2007;57:159-167.

18. Tsukahara M, Nagai H, Kamiakito T, Kawata H, Takayashiki N, Saito K, Tanaka A. Distinct expression patterns of claudin-1 and claudin-4 in intraductal papillary-mucinous tumors of the pancreas. Pathol Int 2005;55:63-69.

19. Chu YW, Yang PC, Yang SC, Shyu YC, Hendrix MJ, Wu R, Wu CW. Selection of invasive and metastatic subpopulations from a human lung adenocarcinoma cell line. Am J Respir Cell Mol Biol 1997;17: 353-360.

20. Miyamori H, Takino T, Kobayashi Y, Tokai H, Itoh Y, Seiki M, Sato H. Claudin promotes activation of pro-matrix metalloproteinase- 2 mediated by membrane-type matrix metalloproteinases. $J$ Biol Chem 2001;276:28204-28211.

21. Morohashi S, Kusumi T, Sato F, Odagiri H, Chiba H, Yoshihara S, Hakamada K, Sasaki M, Kijima H. Decreased expression of claudin-1 correlates with recurrence status in breast cancer. Int J Mol Med 2007; 20:139-143.

22. Cohn ML, Goncharuk VN, Diwan AH, Zhang PS, Shen SS, Prieto VG. Loss of claudin-1 expression in tumor-associated vessels correlates with acquisition of metastatic phenotype in melanocytic neoplasms. J Cutan Pathol 2005;32:533-536.

23. Higashi Y, Suzuki S, Sakaguchi T, Nakamura T, Baba S, Reinecker HC, Nakamura S, Konno H. Loss of claudin-1 expression correlates with malignancy of hepatocellular carcinoma. J Surg Res 2007;139:68-76.

24. Krajewska M, Olson AH, Mercola D, Reed JC, Krajewski S. Claudin-1 immunohistochemistry for distinguishing malignant from benign epithelial lesions of prostate. Prostate 2007;67:907-910.

25. Shih JY, Yang SC, Hong TM, Yuan A, Chen JJ, Yu CJ, Chang YL, Lee YC, Peck K, Wu CW, et al. Collapsin response mediator protein-1 and the invasion and metastasis of cancer cells. J Natl Cancer Inst 2001;93: 1392-1400.

26. Chang CC, Shih JY, Jeng YM, Su JL, Lin BZ, Chen ST, Chau YP, Yang PC, Kuo ML. Connective tissue growth factor and its role in lung adenocarcinoma invasion and metastasis. J Natl Cancer Inst 2004;96:364-375.

27. Weinstat-Saslow DL, Zabrenetzky VS, VanHoutte K, Frazier WA, Roberts DD, Steeg PS. Transfection of thrombospondin 1 complementary DNA into a human breast carcinoma cell line reduces primary tumor growth, metastatic potential, and angiogenesis. Cancer Res 1994;54:6504-6511.

28. Healy KD, Hodgson L, Kim TY, Shutes A, Maddileti S, Juliano RL, Hahn KM, Harden TK, Bang YJ, Der CJ. Dlc-1 suppresses non-small cell lung cancer growth and invasion by RhoGAP-dependent and independent mechanisms. Mol Carcinog 2008;47:326-337. 
29. Goodison S, Yuan J, Sloan D, Kim R, Li C, Popescu NC, Urquidi V. The RhoGAP protein DLC-1 functions as a metastasis suppressor in breast cancer cells. Cancer Res 2005;65:6042-6053.

30. Tobioka H, Tokunaga Y, Isomura H, Kokai Y, Yamaguchi J, Sawada N. Expression of occludin, a tight-junction-associated protein, in human lung carcinomas. Virchows Arch 2004;445:472-476.

31. Younes M, Brown RW, Stephenson M, Gondo M, Cagle PT. Overexpression of GLUT1 and GLUT3 in stage I nonsmall cell lung carcinoma is associated with poor survival. Cancer 1997;80:1046-1051.

32. Donati V, Boldrini L, Dell'Omodarme M, Prati MC, Faviana P, Camacci T, Lucchi M, Mussi A, Santoro M, Basolo F, et al. Osteopontin expression and prognostic significance in non-small cell lung cancer. Clin Cancer Res 2005;11:6459-6465.

33. Michl P, Ramjaun AR, Pardo OE, Warne PH, Wagner M, Poulsom R, D'Arrigo C, Ryder K, Menke A, Gress T, et al. Cutl1 is a target of TGF(beta) signaling that enhances cancer cell motility and invasiveness. Cancer Cell 2005;7:521-532.

34. Chen CN, Hsieh FJ, Cheng YM, Cheng WF, Su YN, Chang KJ, Lee PH. The significance of placenta growth factor in angiogenesis and clinical outcome of human gastric cancer. Cancer Lett 2004;213:73-82.

35. Sawhney RS, Sharma B, Humphrey LE, Brattain MG. Integrin $\alpha 2$ and extracellular signal-regulated kinase are functionally linked in highly malignant autocrine transforming growth factor- $\alpha$-driven colon cancer cells. J Biol Chem 2003;278:19861-19869.

36. Dhawan P, Singh AB, Deane NG, No Y, Shiou SR, Schmidt C, Neff J, Washington MK, Beauchamp RD. Claudin-1 regulates cellular transformation and metastatic behavior in colon cancer. J Clin Invest 2005; 115:1765-1776.

37. Leotlela PD, Wade MS, Duray PH, Rhode MJ, Brown HF, Rosenthal DT, Dissanayake SK, Earley R, Indig FE, Nickoloff BJ, et al. Claudin-1 overexpression in melanoma is regulated by $\mathrm{PKC}$ and contributes to melanoma cell motility. Oncogene 2007;26:3846-3856.

38. Oku N, Sasabe E, Ueta E, Yamamoto T, Osaki T. Tight junction protein claudin-1 enhances the invasive activity of oral squamous cell carcinoma cells by promoting cleavage of laminin-5 $\gamma 2$ chain via matrix metalloproteinase (MMP)-2 and membrane-type MMP-1. Cancer Res 2006;66:5251-5257.

39. Rahner C, Mitic LL, Anderson JM. Heterogeneity in expression and subcellular localization of claudins $2,3,4$, and 5 in the rat liver, pancreas, and gut. Gastroenterology 2001;120:411-422.

40. Filner JJ, Ost D. Principles of staging as applied to nonsmall cell lung cancer. Curr Opin Pulm Med 2008;14:287-291.

41. Benson JR, Weaver DL, Mittra I, Hayashi M. The TNM staging system and breast cancer. Lancet Oncol 2003;4:56-60.

42. Xi L, Lyons-Weiler J, Coello MC, Huang X, Gooding WE, Luketich JD, Godfrey TE. Prediction of lymph node metastasis by analysis of gene expression profiles in primary lung adenocarcinomas. Clin Cancer Res 2005;11:4128-4135.

43. Theodorescu D, Sapinoso LM, Conaway MR, Oxford G, Hampton GM, Frierson HF Jr. Reduced expression of metastasis suppressor RhoGDI2 is associated with decreased survival for patients with bladder cancer. Clin Cancer Res 2004;10:3800-3806.

44. Hicks DG, Yoder BJ, Short S, Tarr S, Prescott N, Crowe JP, Dawson AE, Budd GT, Sizemore S, Cicek M, et al. Loss of breast cancer metastasis suppressor 1 protein expression predicts reduced diseasefree survival in subsets of breast cancer patients. Clin Cancer Res 2006;12:6702-6708.

45. Tsai MF, Wang CC, Chang GC, Chen CY, Chen HY, Cheng CL, Yang YP, Wu CY, Shih FY, Liu CC, et al. A new tumor suppressor DnaJlike heat shock protein, HLJ1, and survival of patients with nonsmall-cell lung carcinoma. J Natl Cancer Inst 2006;98:825-838.

46. Hong TM, Chen YL, Wu YY, Yuan A, Chao YC, Chung YC, Wu MH, Yang SC, Pan SH, Shih JY, et al. Targeting neuropilin 1 as an antitumor strategy in lung cancer. Clin Cancer Res 2007;13:4759-4768.

47. GeneChip expression analysis technical manual [Internet]. Santa Clara, CA: Affymetrix, Inc.; 2006 [updated Feb 21, 2008; accessed Dec 3, 2008]. Available from: www.affymetrix.com/support/downloads/ manuals/expression_analysis_technical_manual.pdf 